\title{
Heartbeat reversal after sectioning the dorsal vessel and removal of the brain of diapausing pupae of Manduca sexta (Lepidoptera: Sphingidae)
}

\author{
KAREL SLÁMA \\ Institute of Entomology, Czech Academy of Sciences, Drnovská 507, 16100 Praha 6, Czech Republic; e-mail: slama@entu.cas.cz
}

Key words. Cardiac functions, heartbeat reversal, anterograde heartbeat, retrograde heartbeat, sectioned heart, myogenic heart, decerebrated pupae, Manduca sexta

\begin{abstract}
Reversal of heartbeat was monitored in vivo by noninvasive, multisensor, thermo-cardiographic and pulse-light, optocardiographic techniques. The dorsal vessel was sectioned at the beginning, in the middle and near the end of the abdomen. Changes in the heartbeat were simultaneously monitored in both the disconnected anterior and posterior sections of the heart. The results revealed the existence of a caudal regulatory cardiac centre located in the fused $\mathrm{A}_{7}-\mathrm{A}_{10}$ abdominal segments. Posterior sections, containing this terminal ampulla of the heart always exhibited a more or less normal heartbeat reversal, including both anterograde and retrograde pulsations. This shows that the forward-oriented as well as the reciprocal, backward-oriented peristaltic waves of the heart are both regulated from the posterior regulatory center, without involvement of the cephalic region. The cardiac pulsations in the anterior sections of the heart were paralysed and seriously impaired by the lesions. During the acute phase after the lesions, anterior sections showed only some convulsion-like, unidirectional, backward-oriented peristaltic pulsations of low frequency. Within one or two days after the lesions, isolated anterior sections of the heart developed a subsidiary heartbeat regulation associated with the oscillating, bi-directional peristaltic waves running alternatively, forward and backward in opposite directions.

After a few days, the previously paralysed anterior sections of the heart were able to develop perfectly coordinated patterns of heartbeat reversal. At this time, the two asynchronous heartbeat patterns ran separately in each of the divided sections of the heart. One or two weeks later, reversal of the heartbeat occasionally occurred synchronously along the entire length of the dorsal vessel. Sectioning of the ventral nerve cord, removal of the cephalic nervous system (brain, frontal ganglion, suboesophageal ganglion and the associated nerves) or removal of the fused terminal abdominal ganglionic mass and adjacent caudal nerves, had no effect on the pattern of heartbeat reversal. These facts indicate that the pupal heart of $M$. sexta operates purely myogenically, like the human heart. The myogenic pacemakers of the caudal regulatory cardiac centre (analogous to the atrio-ventricular nodes of the human heart) are autonomous, generating inherent rhythmicity without intervention from the nervous system. Development of subsidiary pacemakers regulating rhythmicity in the lesioned myocardium and restitution of the synchronized contracting integrity between the two disconnected sections of the heart are new cardiological features, which merit further investigation.
\end{abstract}

\section{INTRODUCTION}

Investigations of the dorsal vessel of insects and other arthropods were common topics of many papers and review articles some three decades ago (Krijgsman, 1952; McCann, 1963; Wigglesworth, 1972; Jones, 1977; Miller, 1977, 1985, 1997; Wasserthal, 1996). In spite of the accumulated data, however, the true physiological nature of cardiac regulation in arthropods still remains a matter of debate. In crustaceans (Limulus polyphemus), for example, the heartbeat functions are generally considered to be neurogenic, because of their sensitivity to neuroactive compounds (review by Krijgsman, 1952; Miller, 1997). On the other hand, the most investigated dorsal vessel of insects (Periplaneta americana) does not show regular responses to the common neurogenic agents and functions perfectly well in the absence of all cardiac neurons. It is thus assumed to be myogenic (Miller, 1974, 1979, 1985; Jones, 1977), although the central nervous system can influence the function of the dorsal vessels and diaphragms in some insects (Miller, 1997).

The possibility that an insect heart might also be regulated by neurogenic factors was reopened by work on Bombyx mori (Ai \& Kuwasawa, 1995). According to these authors, the pattern of heartbeat reversal is con- trolled by nerves from the frontal ganglion and the recurrent nervous system. Similar involvement of the nervous system in regulating the heartbeat was also observed in adult Manduca sexta (Dulcis et al., 2001). In this case the regulation was traced to nerves of the fused A7-A8 terminal abdominal ganglion. It was concluded that the reversal of heartbeat in adult Manduca could be regulated simply by two neural pacemakers. One located in the frontal part of the aorta and the other within the neurons of the terminal abdominal ganglion at the opposite end of the body.

A long time ago, in 1669, Malpighi observed that the dorsal vessel of an insect can be cut transversely into a series of small segments and each piece continues to contract independently (Jones, 1977). This fact was later confirmed in a number of insect species. These observations (see Jones, 1977; Miller, 1985), which are strengthened by the recent electrophysiological studies of Markou \& Theophilidis (2000) on Tenebrio molitor, strongly support the concept of a cardiac regulation based on the presence of local myocardial pacemakers, whose firing cause systolic contractions of the heart. However, it is unknown how the contractile activity is regulated and peristalsis controlled. 
In contrast to the abundance data on the effects of drugs from in vitro studies on hearts (Miller, 1969, 1977, 1985, $1997)$ there is a serious lack of physiological and pharmacological data from in vivo studies. In order to get some information on this aspect, non-invasive cardiographic techniques for prolonged multisensor monitoring of the heartbeat were used on diapausing pupae of Manduca sexta (Sláma, 2003), various stages of Drosophila melanogaster (Sláma \& Farkaš, 2005) and adults of Periplaneta americana (Sláma et al., 2005).

In previous studies (Sláma \& Miller, 2001, 2002; Sláma, 2003, 2004) M. sexta proved to be a very convenient and useful model for prolonged recordings of changes in cardiac activity. Pupal and adult stages of many insects exhibit strong extracardiac pulsations in haemocoelic pressure, which seriously disturb or camouflage the heartbeat patterns (Sláma, 1984, 2000; Sláma \& Neven, 2001; Tartes et al., 2002; Sláma \& Farkaš, 2004). The advantage of using diapausing pupae of $M$. sexta for cardiological studies is that strong extracardiac pulsations associated with $\mathrm{CO}_{2}$ release only last for 20 min every 8 to $16 \mathrm{~h}$. A further advantage is the very stable pattern of heartbeat reversal, with regular switchovers between anterograde and retrograde phases every 5 to $10 \mathrm{~min}$. The anterograde and retrograde pulsations are easily identified by the substantially different heartbeat rates. Moreover, the periods of cardiac rest (diastasis) last only a few minutes in contrast to other diapausing insects in which diastasis can last for several hours. Based on previous experience obtained using this material (Sláma \& Miller, 2001; Sláma, 2003, 2004), extensive in vivo cardiological studies on experimentally transected hearts were initiated and the obtained results have been briefly described here.

\section{MATERIAL AND METHODS}

Diapausing pupae of Manduca sexta (Lepidoptera: Sphingidae) were obtained by rearing the larvae on an artificial diet, using short-day diapause inducing photoperiodic conditions $(8 \mathrm{~h}$ light phase). The main stock of diapausing pupae was kept at room temperature. These "unchilled" pupae were used for measurements within 9 months. After this, some of the pupae end diapause and initiate adult development. The heartbeat of developing pupae increases rapidly and shows variable periods of reversal, which cannot be easily standardized.

Surgical treatments were made in Ringer solution, after immobilisation by submerging the pupae in water for $40 \mathrm{~min}$. Removal of the brain, frontal ganglion and recurrent nerve was also made in Ringer solution through a small window incised in the dorsal side of the head region. The fused terminal abdominal ganglionic mass and adjacent caudal nerves were removed through a small epidermal window made in the ventral side of the fused $\mathrm{A}_{7-9}$ sternites. After removal of the ganglia, the epidermal injury was closed by the excised cuticle. Ringer solution was evacuated and the pupae were dried with absorbent paper. The wounds were covered by a drop of melted wax placed on the operated area by means of a platinum wire loop of the type used in thermocautery.

Sectioning of the dorsal vessel was also made in Ringer solution. The cuticle was incised transversally through the middle of the selected tergite, perpendicular to the underlying dorsal vessel. The dorsal vessel was sectioned using a thin, hooked steel needle, which was gently pulled beneath the incision from one side to the other. Care was taken not to insert the needle too deep under the integument, which could damage the epithelium of the gut. After removal of the Ringer solution, the blotted epidermal incision was sealed with melted wax. There was no mortality; successful separation of the two sections of the heart was recognized by immediate paralysis of the anterior section.

The recording of heartbeat reversal in vivo was made by means of two, noninvasive, electrocardiographic methods described by Sláma (2003). The contact thermocardiographic method uses miniature thermistor sensors, which are positioned outside the body, above the pericardial region (see Wasserthal, 1976; Sláma \& Miller, 2001; Sláma, 2003). In principle, the subintegumental movement of haemolymph within the pericardial region produces changes in the electrical properties of the sensors, which is further amplified and recorded. The advantage of this method is that it quantitatively measures the subepidermal changes in the rate of haemolymph flow. The principle of the second, opto-cardiographic method depends on the use of low frequency pulse-light, which is emitted from special LED diodes. The pulse-light is applied to the pericardial region via an optic fibre. The reflected pulse-light, which is modulated by movements of hemolymph or tissues, is transferred via another, outgoing optic fibre to a phototransistor for further amplification and recording (Sláma, 2003). This method enables true, touchfree recordings of heartbeat to be made at several sites along the dorsal vessel. In combination with the use of optical lenses, the recordings of heartbeat can be made from a distance of $1 \mathrm{~cm}$. In these investigations combined recording of heartbeat using both of these methods were used, however, for practical reasons the illustrations are mostly of optocardiographic recordings, which are more instructive.

In most instances we made simultaneous recordings of the heartbeat from two locations: one in the anterior and the other in the posterior section of the disconnected heart. Occasionally, as indicated in the text, we recorded simultaneously from 4 sensors: 2 were placed before and 2 after the lesion. This was sometimes necessary for the unequivocal identification of the forward or backward propagation of the peristaltic cardiac waves. In most instances recordings were initiated a few hours before the sectioning and continued for $24 \mathrm{~h}$ after the treatment. During the first week after the treatment recordings of the heartbeat were made every day, each for several hours. Then recordings were made once a week for two months. The long term investigations were necessary to cover the period of healing and regeneration of the disconnected hearts.

The electrical output signals were recorded on a PC or on a battery of linear recorders (VAREG, Czech Rep.). Evaluation of the results was made individually by comparing the electrocardiographic records. Major emphasis was given to differences between the simultaneously recorded heartbeat from the anterior and posterior sections of the heart. Figures 1 to 12 show only a few selected electrocardiogramms. The number $(n)$ of recordings in each experiment is given in the text or figure captions.

\section{RESULTS}

The dorsal vessel was sectioned at the anterior end (segment $\mathrm{A}_{2}$ ), middle (segment $\mathrm{A}_{4}$ ) and near the end of the abdomen (segment $A_{6}$ ). Changes in the heartbeat were simultaneously recorded in the anterior and posterior sections. In 2000-2001 only the thermographic method was used. Later on, after the invention of the pulse-light, optocardiographic technique in 2003, all recordings were repeated using this new method. The effects of heart sectioning were always most pronounced in the anterior sections of the heart in the first 2 days. These early acute 


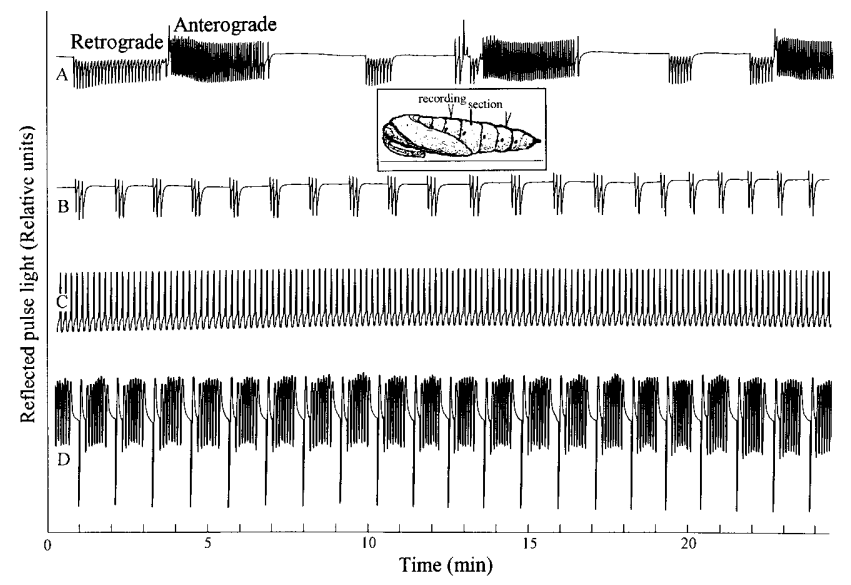

Fig. 1. Optocardiographic recordings of the heartbeat of a diapausing pupa of $M$. sexta, before and after sectioning the dorsal vessel in the 4th abdominal segment (see inset). The recordings were made through the cuticle of the 3rd and 6th (not shown) abdominal tergite at intervals of $2 \mathrm{~h}$ before (A), $2 \mathrm{~h}$ after (B), one day after (C) and two days after sectioning of the heart (D). The upper record (A) shows the normal pattern of heartbeat reversal manifested by the faster, anterograde and the backward-oriented, retrograde phases; $25^{\circ} \mathrm{C}$.

effects of the lesions are thus described separately from the later effects, which were significantly affected by healing of the wounded ends and regeneration of the supplementary regulatory centers of the heart.

\section{Sectioning the heart in the middle of the abdomen (Fig. 1)}

The heart was sectioned in the middle of abdominal tergite $\mathrm{A}_{4}$ in 12 pupae, of which 8 were measured by the contact thermographic and 4 by opto-cardiographic method. The recordings were taken from anterior $\left(\mathrm{A}_{3}\right)$ and posterior $\left(\mathrm{A}_{6}\right)$ segments before the treatment and then at intervals of $2 \mathrm{~h}$ for one day then daily for one week. Each recording lasted for several hours. Subsequent recordings were taken once in a week for two months.

The recording in Fig. 1A shows the common pattern of heartbeat reversal that occurred several minutes before the treatment. It shows the common alternations between the faster anterograde and the slower retrograde phases, with periods of cardiac rest (diastasis). Sectioning of the heart at $\mathrm{A}_{4}$ (see the inset drawing) was associated with the following changes: 1 . There was no effect on the course of heartbeat reversal in the posterior section $\left(\mathrm{A}_{6}\right.$ responses were recorded but not included in Fig. 1 for simplicity); 2. The recording in Fig. $1 \mathrm{~B}$ shows that the heartbeat in the anterior section (taken from $\mathrm{A}_{3}$ ) was seriously impaired. The heartbeat reversal was completely abolished and replaced by a stereotypic, low frequency, double or tripple cardiac convulsions of unknown polarity; 3 . The contractions in the anterior heart portion were completely autonomic, i. e. not synchronized with the normal and continual heartbeat reversal recorded in the posterior section.

It is obvious that disrupting the integrity of the tubular dorsal vessel would seriously affect the functioning of
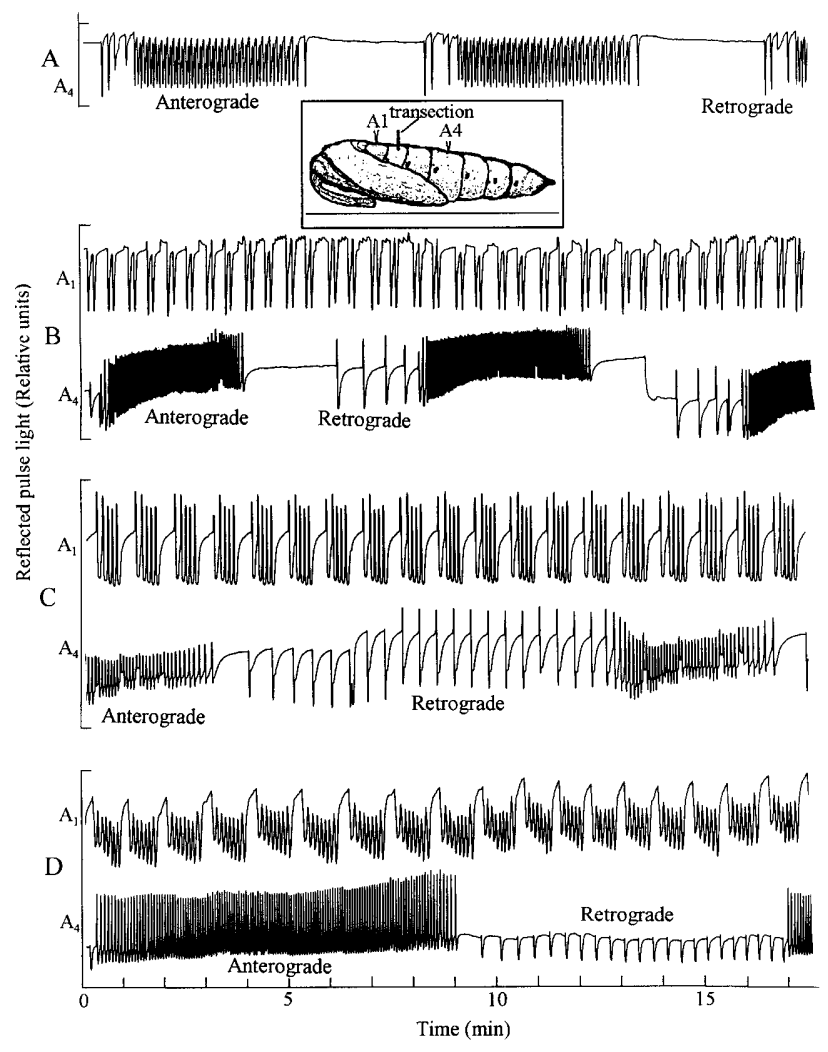

Fig. 2. Optocardiographic recordings of the heartbeat of a diapausing pupa of $M$. sexta before and after sectioning the dorsal vessel in the 2 nd abdominal segment (see inset). The heartbeat was simultaneously recorded in the anterior $\left(\mathrm{A}_{1}\right)$ and posterior $\left(\mathrm{A}_{4}\right)$ parts at intervals of $2 \mathrm{~h}$ before $(\mathrm{A}), 2 \mathrm{~h}$ after $(\mathrm{B})$, one day after (C) and two days after (D) the sectioning. Note that the posterior part preserved normal heartbeat reversal while the anterior part did not; $24^{\circ} \mathrm{C}$.

this organ. The two disconnected parts of the heart probably have open holes at the injured sites. This would have a large impact on the circulation of haemolymph within the body. The effect will be most pronounced in the head and thorax, which normally receive blood only during the anterograde cardiac pulsation. According to the recording in Fig. 1B, however, the disconnected anterior section of the heart failed to show the regular anterograde rhythm.

During the first 2 days after the section, the isolated anterior fragment of the heart underwent specific modulations of the cardiac pulsing. One day after the section there was a more or less constant, stereotypic, low frequency pulsation that can be seen in Fig. 1C. This pattern changed during the next day into a heterogeneous cardiac pulsation (Fig. 1D), with peaks of different amplitude and, as we shall see later, peristaltic waves which went in different directions.

The persistence of apparently normal heartbeat reversal in the posterior portion of transected hearts provides strong presumptive evidence for the presence of a regulatory cardiac centre somewhere within the posterior abdominal metameres of the pupal heart. An important physiological implication of this result would be that the 


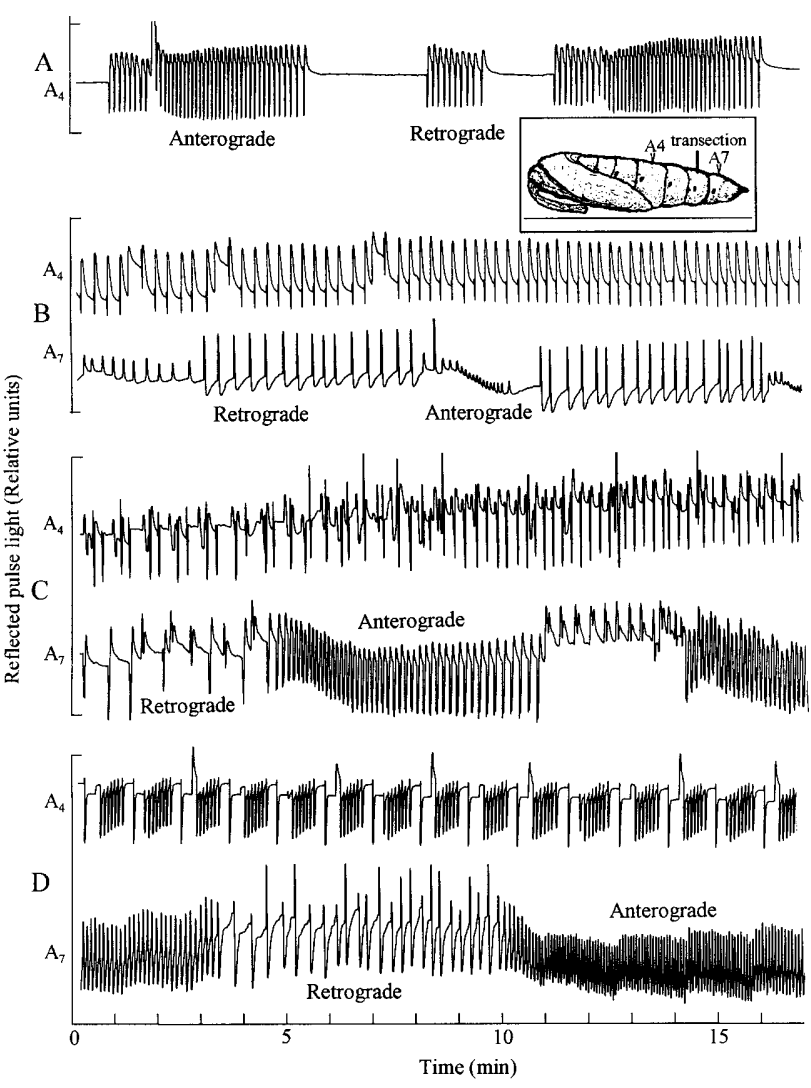

Fig. 3. Similar optocardiographic recording as in Fig. 2, except that the dorsal vessel was sectioned close to the end of the abdomen at $\mathrm{A}_{6}$ (see inset). The heartbeat was simultaneously recorded from the anterior $\left(\mathrm{A}_{4}\right)$ and the posterior $\left(\mathrm{A}_{7}\right)$ parts at intervals of $2 \mathrm{~h}$ before (A), $2 \mathrm{~h}$ after (B), one day after (C) and two days after (D) the treatment. Note that the small terminal fragment $\left(\mathrm{A}_{7}-\mathrm{A}_{9}\right)$ of the dorsal vessel exhibited perfect heartbeat reversal; $24^{\circ} \mathrm{C}$.

heartbeat reversal does not require the presence of a nervous pacemaker in the frontal part of the body. In other words, these results show that the posterior regulatory cardiac centre initiates, under normal physiological conditions, both anterograde and retrograde peristaltic waves. The results obtained with the rest of the pupae were consistent with the results shown in Fig. 1.

\section{Transection of the heart at the proximal end of the abdomen (Fig. 2)}

The heart was sectioned at the beginning of the abdomen $\left(A_{2}\right.$ segment $)$ in 6 pupae, of which 3 were measured using the thermographic and the rest by the optocardiography method. In this case, anterior sections contained only a long narrow tube consisting of the thoracic aorta plus one or two metameres of the heart, whereas the posterior sections possessed a large part of the abdominal heart. The heartbeat was simultaneously recorded in the anterior $\left(\mathrm{A}_{1}\right)$ and posterior $\left(\mathrm{A}_{4}\right)$ abdominal segments. The results of these experiments are collectively illustrated by the recordings in Fig. 2, which are similar to the results shown in Fig. 1. The large posterior sections continued to function normally with regular heartbeat reversal (see Fig. 2; $\mathrm{A}_{4}$ in B, C and D). Shortly

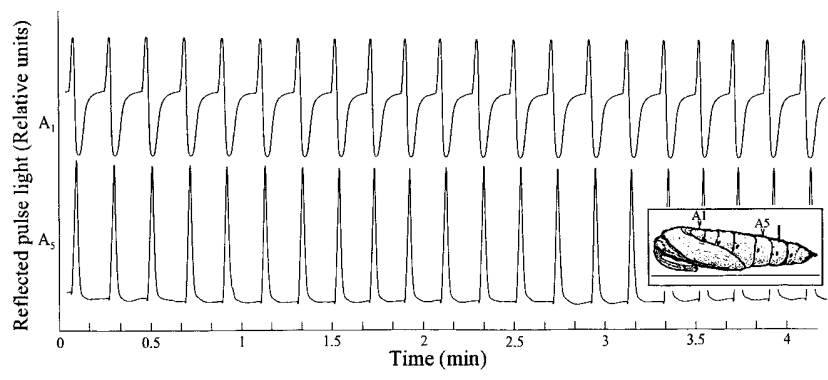

Fig. 4. Simultaneous recording of heartbeat in the anterior $\left(A_{1}\right)$ and terminal part of the abdomen $\left(A_{5}\right)$ one day after sectioning the heart at $\mathrm{A}_{6}$, of a diapausing pupa of $M$. sexta, at $24^{\circ} \mathrm{C}$. The retrograde spikes in $\mathrm{A}_{5}$ occurred with a delay of some $0.6 \mathrm{~s}$.

after sectioning, the anterior section ceased to show a heartbeat. Only a low frequency, convulsion-like, double systolic strokes were recorded. This pathophysiological type of a heartbeat pattern, which never occurred in unoperated pupae, successively developed during the first two days into a specifically modulated rhythm, as shown in Fig. 2; $\mathrm{A}_{1}$ records in $\mathrm{B}, \mathrm{C}$ and $\mathrm{D}$. There was no heartbeat reversal or synchronisation with the posterior section. The results presented in Fig. 2 were similar for all the 6 pupae measured.

\section{Transections of the heart at the distal end of the abdomen (Fig. 3)}

The inset in Fig. 3 shows that the posterior fragment of the heart in this case consisted only of the highly tracheated, terminal heart ampulla, which is located within the fused $\mathrm{A}_{7}$ to $\mathrm{A}_{10}$ abdominal segments. The large anterior fragment thus contained almost the whole pupal heart. The recordings were made simultaneously from the anterior $\left(\mathrm{A}_{4}\right)$ and the posterior $\left(\mathrm{A}_{7}\right)$ abdominal segment in 8 pupae ( 5 by the thermographic and 3 by the optical method).

Curiously enough, this small disconnected terminal fragment of the heart pulsated more or less normally and performed perfect heartbeat reversals (Fig. 3; $\mathrm{A}_{7}$ records in $\mathrm{B}, \mathrm{C}, \mathrm{D})$. This finding indicates that the caudal regulatory cardiac centre is located within the heart ampulla between the fused $\mathrm{A}_{7}-\mathrm{A}_{10}$ abdominal segments at the distal end of the pupa. This newly discovered cardiac regulatory centre initiates regular heartbeat reversal in the absence of the cephalic, thoracic and most of the abdominal dorsal vessel.

Obviously, the normal performance of the small, $\mathrm{A}_{7}-\mathrm{A}_{10}$ fragment was in marked contrast with the paralysis recorded in the bulky anterior section of the heart (see Fig. 3; $\mathrm{A}_{4}$ records in B, C, D). For a few hours after the treatment, the anterior section exhibited a low frequency convulsions, similar to hearts sectioned at $\mathrm{A}_{2}$ and $\mathrm{A}_{4}$. The acute effects of heart sectioning (Figs 1-3) can be summarized as follows: 1. Posterior sections of the heart, containing the terminal $\mathrm{A}_{7}-\mathrm{A}_{10}$ regulatory center retain the ability to pulsate rhythmically and perform heartbeat reversal; 2. Anterior sections disconnected from the terminal $\mathrm{A}_{7}-\mathrm{A}_{10}$ regulatory cardiac centre are para- 


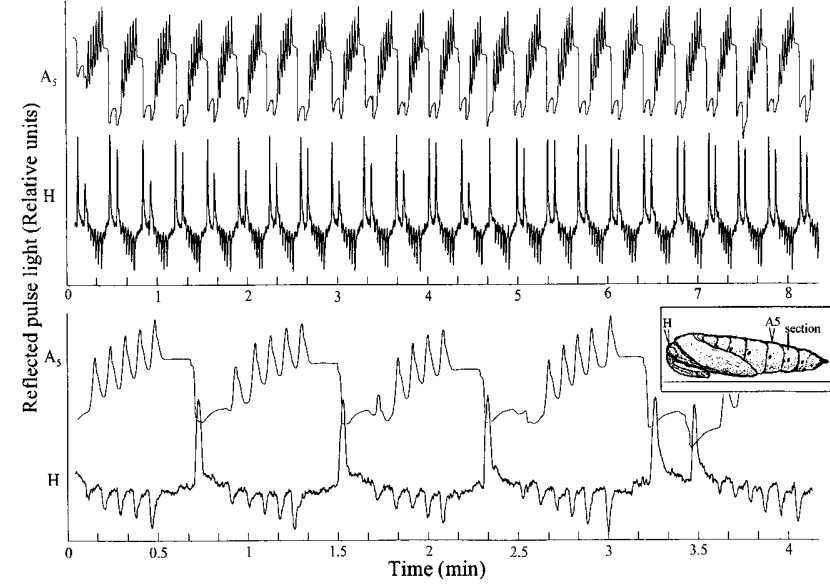

Fig. 5. Simultaneous optocardiographic recordings of the heartbeat in abdominal segment $A_{5}$ and the head $(H) 2$ days after sectioning the heart at $\mathrm{A}_{6}$, of a diapausing pupa of $M$. sexta, at $23^{\circ} \mathrm{C}$. Lower portion is a high resolution recording, which shows retardation of the large anterograde spikes going into the head.

lysed, exhibiting only low frequency convulsions and unidirectional pulsations without reversal, and 3. Within two days of the operation anterior sections of the heart undergo successive restitution of the bipolar, rhythmic cardiac pulsations.

\section{Resumption of heartbeat reversal in anterior sections of the heart (Figs 4-6)}

The uniform, low frequency pathophysiological cardiac pulsations recorded from freshly isolated anterior portions of the heart (see Fig. 1B; Fig. 2B- $\mathrm{A}_{1}$ and Fig. $3 \mathrm{~B}-\mathrm{A}_{4}$ ) were unidirectional. They were similar to spontaneous pulsations of explanted hearts in vitro. However, highresolution optocardiographic recordings from pupae sectioned at $\mathrm{A}_{6}$ revealed that the heart did not contract along its entire length simultaneously. In other words, there was a peristaltic wave that moved backwards, i. e. pulsations recorded immediately after the lesions had retrograde polarity (see Fig. 4). The systolic spikes arrived from segment $A_{1}$ to $A_{5}(18 \mathrm{~mm})$ with a delay of some 0.4 to 0.6 sec. This difference is too small to be clearly distinguished on the time scale in Fig. 4. However, the retrograde orientation of the peristaltic waves was unequivocally determined visually, by the delayed movement of the posterior pen driver $\left(A_{5}\right)$ in the course of the recordings.

Previous investigations revealed that the retrograde pulsations occur predominantly in the abdomen (Sláma \& Miller, 2001). They were insufficient to insure satisfactory haemolymph circulation within the head. A pupa with an exclusively retrograde heartbeat, such as the one in Fig. 4 after the operation, would sooner or later suffer from reduced circulation of blood within its head. This does not happen, however, as certain peristaltic cardiac waves become diverted in the opposite direction and start pumping haemolymph into the head. This process of successive transformation of unipolar peristaltic waves into

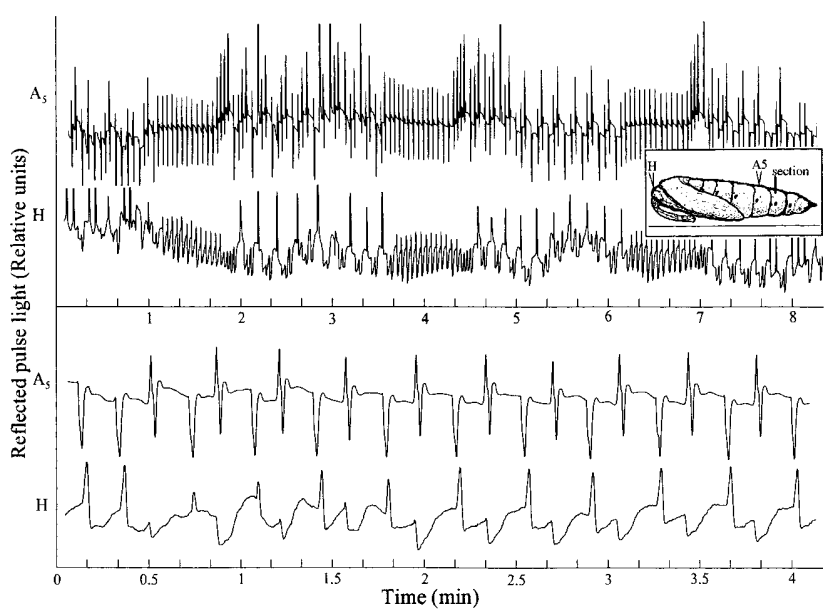

Fig. 6. Similar optocardiographic recordings as in Fig. 5 obtained using another pupa of M. sexta 2 days after sectioning its heart. The records show complete resumption of the heartbeat reversal in the anterior fragment of the heart, dissociated from the terminal regulatory center.

bipolar ones occurred within $24 \mathrm{~h}$ in all isolated anterior sections and is documented in Figs 1D, 2D, 3D and 4 by the different amplitude and frequency of the systolic cardiac contractions. Moreover, the recordings in Fig. 5 document the ability of the pupal circulatory system to adjust to the deficiency produced by the lesion. They show a new pattern of heartbeat reversal 2 days after separation of the anterior section. After the lesion the originally unidirectional pulses are replaced by regular switchovers between anterograde and retrograde peristalsis (see Fig. 5). The polarity of the peristalsis is easily identified by the reciprocal directions of the systolic peaks and especially the delay in propagation of the peristaltic waves between $\mathrm{A}_{5}$ segment and the head. The pupa used in Fig. 5 actually alternated some 5-6 retrograde pulses with one or two reciprocal, stronger anterograde pulses. The recordings in Fig. 5 provide a good example of a previously paralysed anterior portion of the heart developing after some time a subsidiary peristaltic rhythmicity with the waves alternatively running in opposite directions. The physiological implication of this reversal is obvious: increased haemolymph circulation at both ends, i. e. within the head as well as at the cut end of the tubular heart.

The resumption of heartbeat reversal occurred in all isolated anterior sections (sections at $\mathrm{A}_{2}, \mathrm{~A}_{4}$, and $\mathrm{A}_{6}$ ). The records in Figs 1-5 contain several examples of the resumption of heartbeat reversal. Using the noninvasive, touch-free, pulse-light technique several uninterrupted recordings were made over the 2-day period after sectioning. Evaluation of these records revealed that the first signs of the resumed bipolar peristalsis occurred between 16 to $23 \mathrm{~h}$ post treatment (at $23^{\circ} \mathrm{C}$ ). It started usually as oscillating, "up-and-down", individual pulses of reciprocal polarity. Further modifications included alternations of several retrograde pulses with one or just a few anterograde strokes, as shown in Fig. 5. 


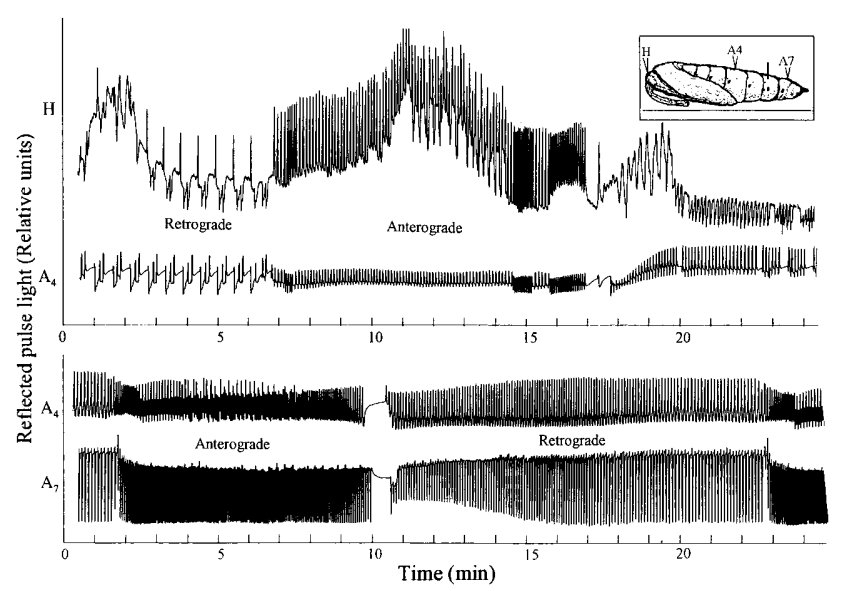

Fig. 7. Simultaneous optocardiographic recordings of heartbeat recorded between the head and $\mathrm{A}_{4}$ and between segments $\mathrm{A}_{4}$ and $\mathrm{A}_{7}$ one week after transection of the dorsal vessel in $\mathrm{A}_{6}$ (see inset). These records were for the same pupa as used for Fig. 3. They show resumption of the synchronised heartbeat reversal over the entire length of the dorsal vessel. Note persistence of the "up-and-down", bidirectional oscillations during the retrograde phase.

The high resolution, simultaneous recordings between the head and $\mathrm{A}_{5}$ abdominal segments (see the bottom section of Fig. 5) were made using pupae sectioned at $A_{6}$. In this case, all isolated anterior fragments resumed an independent heartbeat reversal within 2 days. In certain cases, such as the pupa measured in Fig. 6, normal heartbeat reversal was restored well before the 2 nd day after sectioning. The records in Fig. 6 also show the persistence of the emergency, "up-and-down" bipolar heartbeat patterns between the bouts of more or less consolidated unidirectional anterograde activity. In all such recordings from the head region, the retrograde pulsations had extremely small amplitude or were completely absent, which enabled them to be identified. An important physiological implication of the above is that the pupal dorsal vessel is able to reconstitute and repair itself after injury.

\section{Synchronization between the anterior and posterior sections of the heart (Figs 7-12)}

In further experiments the future fate and possible resumption of synchronization between pulsations in the anterior and posterior sections of the heart were investigated. It can be anticipated that disconnected sections of the heart undergo a complex of physiological changes associated with healing and regeneration of the injured myocardium. Thus, the cardiological investigations had to be extended for several weeks, in some cases even for two months. The most important physiological changes, however, occurred within one or two weeks of the lesions. There follows only a brief account of the basic results, with a few selected examples of the authentic records for easier understanding of the text (see Figs 7-12).

The first and most consistent synchronization of the heartbeat reversal between the anterior and posterior sections occurred in all 8 pupae sectioned at the distal end of

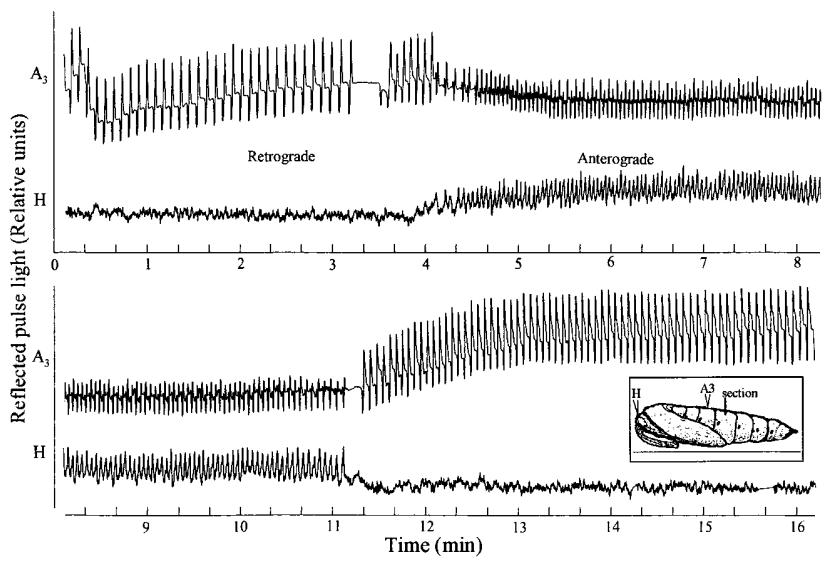

Fig. 8. Simultaneous optocardiographic recordings from the head and $\mathrm{A}_{3}$, which show full restoration of the heartbeat reversal in the anterior section of the heart one week after sectioning of the dorsal vessel at $\mathrm{A}_{4}$ (see inset). Note restoration of the well expressed periods of reciprocal pulsations and the absence of retrograde peristalsis in the head.

the abdomen $\left(\mathrm{A}_{6}\right)$. The records in Fig. 7 show synchronization across the entire length of the dorsal vessel one week after the lesion in one of these pupae. The heart of this pupa was indeed disconnected by the lesion, which is documented by the records obtained earlier from the same pupa (cf. Fig. 7 and Fig. 3).

The group of 12 pupae sectioned in the middle of the abdomen $\left(\mathrm{A}_{4}\right)$ did not yield such uniform results. In this group, 8 pupae restored the anterior-posterior synchronisation of the heartbeat within 1 to 3 weeks (see records in Fig. 8 and 9). The remaining 4 pupae showed perfect heartbeat reversal in the previously paralysed anterior sections, but had not resumed the anterior-posterior synchronisation after two months (see Fig. 10). Here the lack of sufficient hemolymph circulation in the bulky anterior part of the body was periodically repaired by strong extracardiac contractions, which were produced by intersegmental muscles of the abdominal pressure pump (= false heartbeat, see arows in Fig. 10). Peaks of strong extracardiac pulses were also recorded by the thermographic method (not shown in Fig. 10). This method revealed changes in the subintegumental flow of haemolymph. Extremely large peaks recorded by this method during the extracardiac haemocoelic pulsations confirmed their association with the increased haemolymph circulation in the anterior part of the body. Thus, a long-lasting cardiac insufficiency in haemolymph circulation can be compensated for by strong extracardiac pulsations of neurogenic origin.

The pupae with the heart sectioned at the proximal end of the abdomen $\left(\mathrm{A}_{2}\right)$ never resumed the anterior-posterior synchronisation of the heartbeat. They developed extensive anterograde pulsations in the anterior section (see upper part in Fig. 11), but this was never synchronized with the anterograde pulsation in the large posterior section (Fig. 11, lower part). In two out of 6 pupae, there was only a partial or incomplete synchronisation. In this case, as shown in Fig. 12, the periods of anterograde pul- 


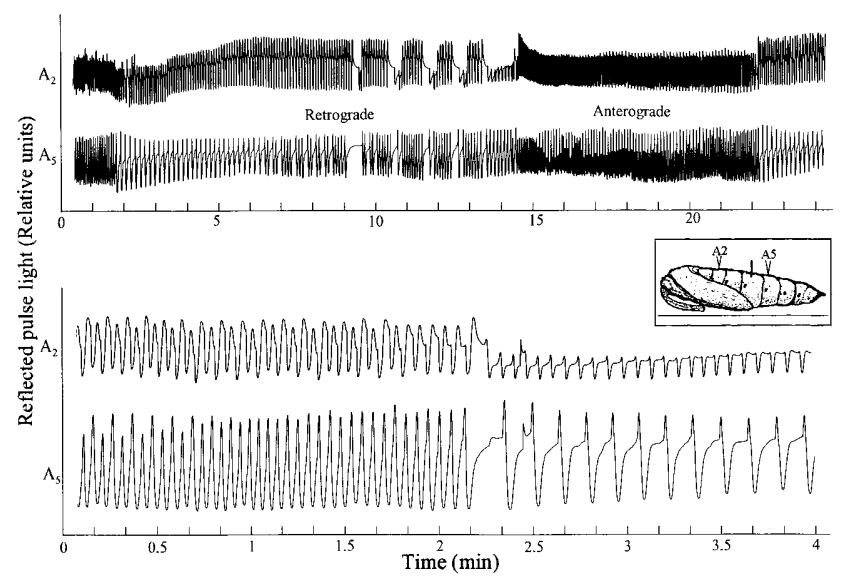

Fig. 9. Simultaneous optocardiographic recordings from the anterior $\left(\mathrm{A}_{2}\right)$ and posterior $\left(\mathrm{A}_{5}\right)$ sections of the heart of the same pupa used for Fig. 8, which show full resumption of the synchronised heartbeat reversal between the two originally disconnected parts of the dorsal vessel.

sation occurred usually during similar, but not exactly the same periods. However, the individual systolic contractions showed only partial synchronization (Fig. 12, above), or were asynchronous (Fig. 12, below). In summary, the easiest anterior-posterior synchronization occurred in pupae sectioned at $\mathrm{A}_{6}$, less frequent synchronization in pupae sectioned at $\mathrm{A}_{4}$, whereas a virtual lack of synchronization was found in those sectioned at $\mathrm{A}_{2}$. It seems, therefore, that the ability to reconstitute synchronization depends, among other things, on the size of the isolated anterior section. The presence of synchronization suggests that the two pieces of the dorsal vessel were reconnected by regeneration. This view is favoured by the delay of several days, which would be sufficient time for regeneration and physical reunification of the myocardium.

\section{Removal of the brain and the fused terminal abdominal ganglion}

So far, there are no well documented physiological reasons for the direct involvement of the nervous system in cardiac regulations in insects. The idea of neurogenic pacemakers at opposite ends of the body, initiating the waves of reciprocal heart peristalsis, turns out to be unrealistic or illusory. Nevertheless, the possible role of the integrity of the nerve cord was tested by cutting the cord between the $\mathrm{A}_{4}$ and $\mathrm{A}_{5}$ ganglia in 3 diapausing pupae of Manduca. These lesions did not affect heartbeat significantly compared to 3 sham operations. This shows that the forward or backward propagation of the peristaltic cardiac contractions in vivo does not depend on the integrity or conductivity of the ventral nerve cord. Furthermore, the whole anterior nervous complex consisting of the brain, frontal ganglion and suboesophageal ganglion with the adjacent nerves $(n=5)$ were carefully extirpated. In addition, the fused abdominal ganglionic complex $(n=4)$ with the adjacent nerves at the opposite end of the body were also removed. The heartbeat reversal of these surgically treated pupae was monitored

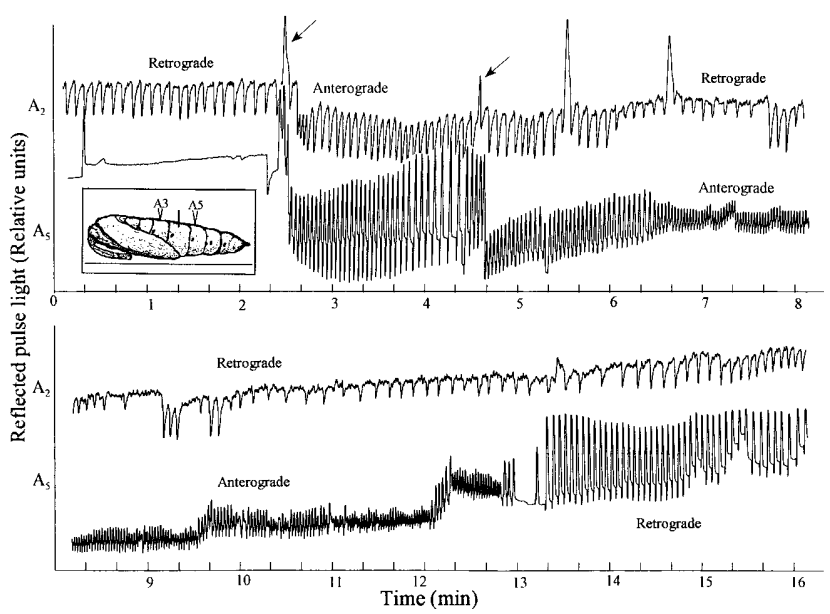

Fig. 10. Simultaneous optocardiographic recordings from the anterior $\left(\mathrm{A}_{2}\right)$ and posterior $\left(\mathrm{A}_{5}\right)$ sections of the heart of a diapausing pupa of $M$. sexta one month after transection of the dorsal vessel at $\mathrm{A}_{4}$. The anterior section developed a heartbeat reversal that is not synchronized with that recorded from the posterior portion. The circulation of haemolymph throughout the anterior part of the body was periodically increased by vigorous extracardiac pulses (arrows), which are visible as large spikes on the records.

by the thermographic method at intervals from one day before to $5 \mathrm{~h}, 1,5,14$ and 30 days afterwards. The results revealed that neither removal of the brain nor the terminal ganglionic mass of the abdomen exerted any short- or long-term influence on the steady pattern of heartbeat reversal in diapausing pupae. It is not necessary to illustrate these negative results by further figures. It is important, however, to point out that these negative results rule out any possibility that regulation of heartbeat reversal in pupae of $M$. sexta is controlled by a nervous pacemaker at each end of the body. It may be also worth stating that functions of the terminal $\mathrm{A}_{7}-\mathrm{A}_{10}$ cardiac regulatory centre were also unaffected by extirpations of essential components of the central nervous system. These experiments clearly reveal that the pupal heart of $M$. sexta operates on purely myogenic principles.

\section{DISCUSSION}

The following quotation from the book by Jones (1974, pp. 44-51) recapitulates our level of understanding of insect cardiology: 1."In numerous insects, heartbeat begins at the posteriormost end of the vessel, and a succession of contraction waves pass toward the head; 2 . In a number of species, aorta is elastic but not actively contractile, although in other insects both the heart and aorta contract; 3 . The anterior aorta can beat at an entirely different rate and manner from the posterior heart; 4 . Throughout larval life, the vessel pulsates only in a forward direction and cannot be induced to pulsate backwards; 5. The pupal heart readily shows backward, bipolar, and sometimes central beating; 6 . The anteriorly directed contractions are usually rapid, and the posterior waves slow". These characteristic features of the insect dorsal vessel are also true for $M$. sexta, although recorded 


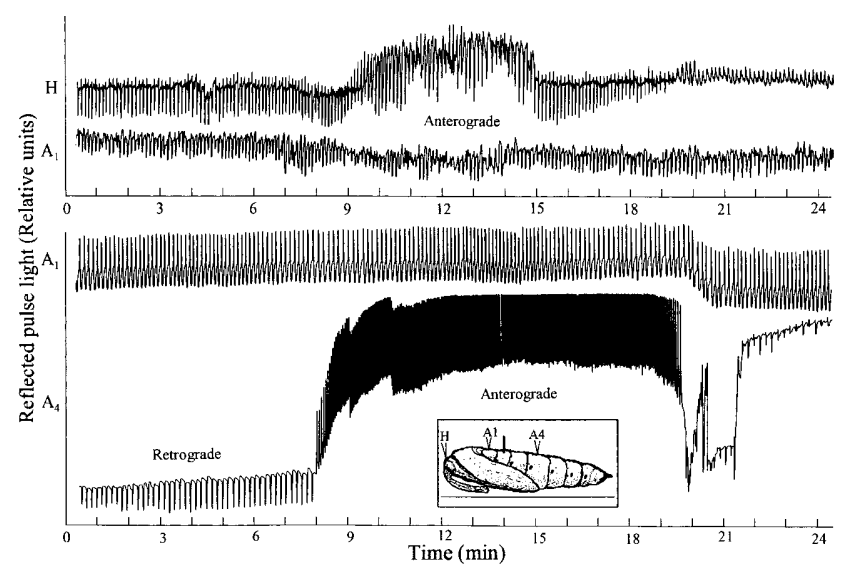

Fig. 11. Optocardiographic records of the heartbeat in the anterior (above) and between the anterior $\left(\mathrm{A}_{1}\right)$ and posterior $\left(\mathrm{A}_{4}\right)$ sections of the heart (below), 14 days after sectioning in $\mathrm{A}_{2}$ the dorsal vessel of a diapausing pupa of $M$. sexta, at $25^{\circ} \mathrm{C}$. Note the presence of anterograde pulsations of different amplitude in the head (above) and the absence of anterior-posterior synchronisation (below).

visually and with a stopwatch a quarter of a century ago (for more recent reviews see Miller, 1985, 1997). The pupal heartbeat of $M$. sexta was more recently investigated by Smits et al. (2000), using an old, invasive impedance conversion method with implanted wire electrodes. They did not record any discernible rhythm in heart contractions in the pupal stage and concluded that "oscillations in the direction of the heart contraction ('reversals') were common but unpredictable". However, Sláma and Miller $(2001,2002)$ subsequently described very constant and perfectly coordinated cycles of heartbeat reversal in these pupae, which were used for investigating cardiac regulation in insects (Sláma, 2003, 2004). In addition to the regular switchover between the bipolar pulsations, the pupal heart of $M$. sexta exhibits special modifications of the unidirectional cardiac rhythms, usually in the form of intercallated cardiac pulses, which start in the middle of the body (Sláma \& Miller, 2001; Sláma, 2003).

Studies on the functioning of transected hearts are not new. Malpighi used this technique in his pioneering work in 1669 (see the quotations in Jones, 1977). Yaeger (1939) observed that a single isolated chamber of the heart of Periplaneta can pulsate rhythmically and with normal amplitude. In a semi-isolated preparation of the heart of Tenebrio molitor, the isolated anterior section continued to pulsate with a similar frequency to the posterior section (Markou \& Theophilidis, 2000). This led these authors to conclude that the cardiac pacemakers could be located along the whole heart. Experiments in vivo on Locusta, on the other hand, revealed that the anterior section of a transected heart always pulsated more slowly than the posterior section (Roussel, 1971). The results presented here clearly show that the difference in cardiac function between the anterior and posterior portions of transected hearts can be substantial. The contracting of the anterior portion of the heart was paralysed

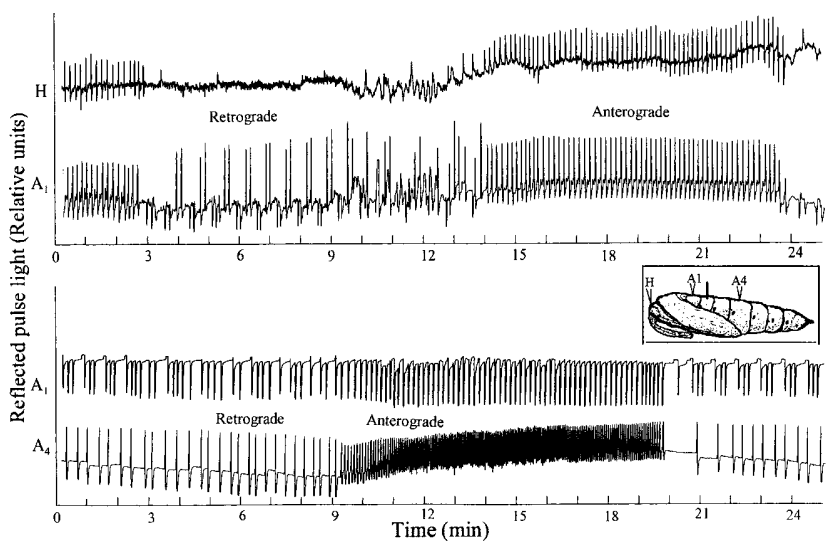

Fig. 12. Optocardiographic records of the heartbeat in the anterior (above) and between the anterior and posterior sections (below), one month after sectioning in $\mathrm{A}_{2}$ of the dorsal vessel of a diapausing pupa of $M$. sexta, at $25^{\circ} \mathrm{C}$. Note the presence of perfectly resumed anterograde pulsations in the head (above) and incompletely resumed cardiac synchronisation between the anterior and posterior portions (below).

for several hours, whereas the posterior section continued to pulsate more or less normally in both directions. The finding of the essential regulatory cardiac centre in the terminal ampulla of the heart in pupae of $M$. sexta certainly merit further investigation, because it shows certain analogies with the regulatory, atrio-ventricular nodus of the human heart (Katz, 1992). It was suggested by Tartes et al. (2002) that cycles of heartbeat activity in many insects may be regulated by extracardiac movements of the abdomen. In our experiments with the pupae of $M$. sexta, however, there was no causal relationship between the myogenic patterns of heartbeat reversal and neurogenic abdominal contractions.

According to Jones (1977), hearts that normally beat cyclically in a forward and backward direction have a pacemaker at each end of the tube. This rather old and superficially logical physiological view was never supported by experimental evidence. It was claimed more recently for the heart of adult $M$. sexta by Dulcis et al. (2001). In pupae of the same species, not only the anterograde, but also the reciprocal retrograde peristaltic pulsations actually start at the caudal end of the dorsal vessel (Sláma \& Miller, 2001; Sláma, 2003). This suggests that the existence of a cardiac pacemaker at each end of the body has no physiological justification. We have now confirmed that a single posterior regulatory cardiac centre is positively able to initiate both the forward and backward directed peristaltic cardiac waves. The best example of such highly autonomic regulation can be found in the heartbeat reversal in quite small, $\mathrm{A}_{7}-\mathrm{A}_{9}$ terminal fragments of the heart (see Fig. 3). This finding is new and not previously recorded for insects. Another new fact is that the frontal nerve ganglia (brain and associated cephalic ganglia) or the caudal nerve ganglia (fused terminal ganglia with the associated nerves) at the other end of the body do not play any significant role in the physiological regulation of heartbeat reversal. 
Previous views concerning the pacemaker activity of insect hearts were reviewed earlier by Miller (1985). It is suggested (Miller, 1997) that the insect central nervous system can exert a nervous control over the dorsal vessels and diaphragms. According to our opinion, however, this suggestion can only apply to the heart of the American cockroach, Periplaneta americana, which is innervated and exhibits a number of abnormal cardiological features that are germane to other insect species (Sláma et al., 2006). Existence of neurogenic cardiac pacemakers mediated by cephalic (Ai \& Kuwasawa, 1995) or caudal (Dulcis et al., 2001) nerves is in conflict with the present findings, which rule out the involvement of the nervous system in regulation of the heartbeat reversal. In contrast, the results presented here support Jones (1977) and Markou \& Theophilidis (2000), who conclude that the cardiac pacemakers are myogenic and reside within the myocardium of the heart.

By using noninvasive electrocardiographic methods heartbeat recordings were obtained in vivo over periods of hours, days or weeks. These long-term recordings revealed the enormous regeneration potential of the insect dorsal vessel. The development of subsidiary patterns of heartbeat reversal in the previously paralysed anterior section reveals the potential of insect hearts to recover heartbeat rhythmicity after the connections to the posterior regulatory center were cut. This is again reminiscent of the subsidiary pulsation rhythmicity in the human heart after the disabling of the atrio-ventricular nodus (Katz, 1992). The availability of prolonged, in vivo cardiographic recordings for insects may open new avenues for comparative physiological studies and also for pharmacological investigations of cardioactive compounds in vivo (Sláma, 2004; Sláma \& Rosinski, 2004; Sláma et al., 2006). So far, our knowledge of insect cardioactive compounds is almost exclusively based on investigations in vitro (see Miller, 1979, 1985, 1997 for review). It appears, however, that the results obtained from explanted hearts in vitro are substantially different from those obtained using whole insects (Sláma, 2004). The methods described in this paper are currently being used in extensive pharmacological investigations to determine similarities with the pharmacological data for the human heart.

ACKNOWLEDGEMENTS. We are greatly indebted to T.A. Miller, Department of Entomology, University of California, Riverside, CA, for extensive advise and helpful criticism of the text. We also wish to acknowledge with thanks the corrections to the text made by S. Phillips, San Francisco, CA and the supply of diapausing pupae of $M$. sexta by L.M. Riddiford, University of Washington, Seattle, WA, USA.

\section{REFERENCES}

Ai H. \& Kuwasava K. 1995: Neural pathways for cardiac reflexes triggered by external mechanical stimuli in larvae of Bombyx mori. J. Insect Physiol. 41: 1119-1131.

Dulcis D., Davis N.T. \& Hildebrand J.G. 2001: Neuronal control of heart reversal in the hawkmoth Manduca sexta. $J$. Comp. Physiol. (A) 187: 837-849.
Jones J.C. 1977: The Circulatory System of Insects. Ch.C. Thomas Publisher, Springfield, IL, 255 pp.

Katz A.M. 1992: Physiology of the Heart. 2nd ed. Raven Press, New York, 613 pp.

KriJgSman B.J. 1952: Contractile and pacemaker mechanisms of the heart of arthropods. Biol. Rev. Cambridge Philos. Soc. 27: 320-346.

Markou T. \& Theophilidis G. 2000: The pacemaker activity generating the intrinsic myogenic contraction of the dorsal vessel of Tenebrio molitor (Coleoptera). J. Exp. Biol. 203: 3471-3483.

MC CANN F.V. 1963: Electrophysiology of an insect heart. $J$. Gen. Physiol. 46: 803-821.

MilLER T.A. 1969: Cockroach heart response to cardioaccelerators. Entomol. Exp. Appl. 12: 53-61.

MiLLER T.A. 1979: Nervous versus neurohormonal control of insect heartbeat. Am. Zool. 19: 77-86.

Miller T.A. 1985: Structure and physiology of the circulatory system. Comp. Insect Physiol. Biochem. Pharmacol. 3: 289-353.

Miller T.A. 1997: Control of circulation in insects. Gen. Pharmacol. 29: 23-38.

Roussel J.P. 1971: Le rythme cardiaque de Locusta migratoria L. et ces variations au cours du développement larvaire. $C$. $R$. Acad. Sci. (D) 273: 1586-1589.

SLÁmA K. 1984: Recording of haemolymph pressure pulsations from the insect body surface. J. Comp. Physiol. (B) 164: 635-643.

SLÁma K. 2000: Extracardiac versus cardiac haemocoelic pulsations in pupae of the mealworm (Tenebrio molitor L.). $J$. Insect Physiol. 46: 977-992.

SLÁmA K. 2003: Mechanical aspects of heartbeat reversal in pupae of Manduca sexta. J. Insect Physiol. 49: 645-657.

SLÁMA K. 2004: The effect of Corazonin on heartbeat reversal in pupae of the tobacco hornworm, Manduca sexta. Eur. J. Entomol. 101: 513-521.

SLÁma K. \& FARKAŠ R. 2005: Heartbeat patterns during the postembryonic development of Drosophila melanogaster. $J$. Insect Physiol. 51: 489-503.

Sláma K. \& Miller T.A. 2001: Physiology of heartbeat reversal in diapausing pupae of the tobacco hornworm, Manduca sexta (Lepidoptera: Sphingidae). Eur. J. Entomol. 98: 415-431.

SLÁMA K. \& Miller T.A. 2002: Heartbeat reversal in pupae of the tobacco hornworm (Manduca sexta). In Konopińska D. (ed.): Arthropods - Chemical, Physiological and Environmental Aspects. Wydawnictwo Uniwersitetu Wroclawskiego, Wroclaw, pp. 27-34.

SlÁmA K. \& Neven L. 2001: Active regulation of respiration and circulation in pupae of the codling moth (Cydia pomonella). J. Insect Physiol. 47: 1321-1336.

SLÁMA K. \& RosińSKI G. 2004: Delayed pharmacological effects of proctolin and CCAP on heartbeat in pupae of the tobacco hornworm, Manduca sexta. Physiol. Entomol. 30: 14-28.

Sláma K., SAKai T. \& TaKeda M. 2006: Effect of corazonin and CCAP on heartbeat in the American cockroach (Periplaneta americana). Arch. Insect Physiol. Biochem. (in press).

Smits A.W., Burggren W.W. \& Oliveras D. 2000: Developmental changes in in vivo cardiac performance in the moth Manduca sexta. J. Exp. Biol. 203: 369-378.

Tartes U., Vanatoa A. \& KuUsik A. 2002: The insect abdomen - a heartbeat manager in insects. Comp. Biochem. Physiol. (A) 133: 611-623.

WASSERTHAL L.T. 1976: Heartbeat reversal and its coordination with accessory pulsatile organs and abdominal movements in Lepidoptera. Experientia 32: 577-578. 
Wasserthal L.T. 1996: Interaction of circulation and tracheal ventilation in holometabolous insects. Adv. Insect Physiol. 26: 297-351.

Wigglesworth V.B. 1972: The Principles of Insect Physiology. 7 th ed. Chapman \& Hall, New York, 827 pp.
YAEGER J.F. 1939: Electrical stimulation of isolated heart preparations from Periplaneta americana. J. Agric. Res. 59: 121-137.

Received May 26, 2005; revised and accepted August 3, 2005 